\title{
Conservaciones e invariantes cognitivos en el desarrollo. Aspectos psicológicos y epistemológicos
}

\section{Vicente Bermejo}

Universidad Complutense

\section{INTRODUCCION}

Los primeros estudios de la escuela de Ginebra en torno a las conservaciones aparecen al final de los años treinta e inicios de la década siguiente (Inhelder, 1936; Piaget e Inhelder, 194I; Piaget y Szeminska, 1941). Sin embargo, la literatura psicológica apenas presta atención al tema hasta los años sesenta y, sobre todo, durante el último decenio, en que se multiplican considerablemente las publicaciones y se polemiza con gran vivacidad sobre los determinantes de esta noción, así como sobre la posibilidad de su adquisición mediante aprendizaje, sobre su precocidad y posible extinción (Acredolo, 1981, 1982; Bovet y otros, 1981; Brainerd, 1979, 1982; Bruner, I966; Conchillo, I 982, I 983 ; Cowan, 1979; Donaldson, 1982 ; Field, 198 1; Hargreaves y otros, 1982; Hunt, I975; Kanno, 1979; Light y otros, I979; McGarrigle y Donaldson,
1976; Miller, 1982; Murray, 1981; ParratDayan y Bovet, 1982; Piaget e Inhelder, 1941, 1963; Piaget y Szeminska, 1941; Russell, 1979; Schultz y otros, 1979; Silverman y Rose, 1982; Wheldall y Poborca, I980, etc.).

A pesar de esta abundancia bibliográfica, de la que sólo hemos referido una muy reducida muestra, y de la pequeña, pero relativamente larga historia del tema, continúan las discrepancias entre los autores promotores de esta animada controversia (ver, por ejemplo, Acredolo, 1982; Bovet y otros, 1981; Conchillo, I983). Con este contexto de fondo, el presente trabajo persigue el doble objetivo de pergeñar algunas puntualizaciones, tanto de carácter psicológico como epistemológico, que clarifiquen en la medida de lo posible las diferentes opciones adoptadas por los autores, y de fijar en consecuencia posiciones a la luz explicativa de las aclaraciones dibujadas.

Dirección: Colonia Sta. Teresa, I. Pozuelo de Alarcón. Madrid. 


\section{LOS INVARIANTES A LO LARGO DEL DESARROLLO}

La mejor metodología, tomado el término en su genuina etimología griega, para descubrir el sentido profundo de las nociones de conservación, consiste, a nuestro juicio, en situar dicha noción dentro del marco evolutivo, como un eslabón más que constituye y fundamenta la secuencia del desarrollo, analizando sus antecedentes más significativos y avanzando, en la medida de lo posible, la línea evolutiva posterior. En esta óptica, el desarrollo infantil aparece jalonado por un conjunto de invariantes cognitivos que modulan profundamente su comportamiento, tal como reseñamos en un trabajo anterior (Bermejo, 1982 a), enfatizando la importancia de estos invariantes, juntamente con la presencia indiscutible del cambio, a lo largo del desarrollo. Las conservaciones constituyen uno de estos invariantes evolutivos, que aparecen un tanto tardíamente (hacia los seis o siete años, según los autores), pero que, de algún modo, se prefiguran conceptualmente en otros más precoces, algunos de los cuales revisamos a continuación.

\section{Las constancias perceptivas}

El primero de estos invariantes son las constancias perceptivas (forma, tamaño, color, etc.), mediante las cuales el niño llega a percibir la forma aproximadamente exacta de un objeto (o su tamaño), a pesar de los cambios de perspectiva y distancia de dicho objeto con respecto al ojo del observador. Más en concreto, y con respecto a la constancia visual del tamaño, las estimaciones emitidas por el niño llegan a ser prácticamente constantes, a pesar de las variaciones producidas en el ángulo visual y, en consecuencia, en su imagen retiniana, a medida que se modifica la distancia existente entre el ojo y el objeto-estímulo. $Y$ esta constancia llega incluso a conservarse, con pequeñas variaciones, cuando las estimaciones se llevan a cabo en un laboratorio de cuarenta metros de distancia, por niños de edad superior a los seis años (Bermejo, I 98 I a).

En la literatura sobre este fenómeno perceptivo aparecen, a nuestro juicio, dos líneas de trabajo claramente diferentes, e incluso una tercera, que resulta un tanto ambigua al sufrir probablemente la doble influencia de las posiciones anteriores. La primera orientación es de corte empirista, apareciendo claramente definida en el clásico trabajo de Halways y Boring (I94I) y posteriormente se transmite a través de los estudios defensores de la hipótesis de la invarianza tamaño/distancia (Gilinski, I95 I; Kilpatrick e Ittelson, I953; Kuroda, 1963 , etc.). Siguiendo de cerca los postulados epistemológicos positivistas y llevados probablemente por un excesivo celo experimentalista, Boring (1940) y Halways y Boring (194I) proponen un paradigma de investigación más adecuado para analizar la ley de Emmert (188I) que para estudiar la constancia visual del tamaño. Según este modelo (ver fig. I), el ángulo visual permanece constante,

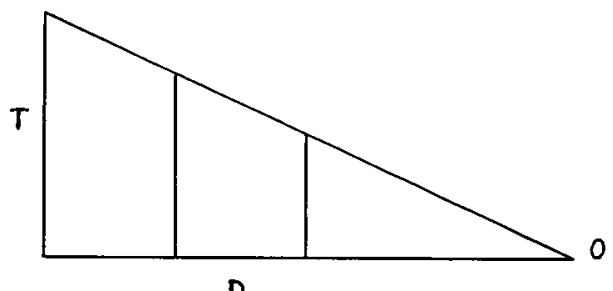

D

Fig. 1.-T: tamaño de los estimulos; $D$ : distancia física, y O: ojo del observador.

variando proporcionalmente la distancia y el tamaño de los estímulos. En consecuencia, el grado de constancia vendría dado por el grado de exactitud de las respuestas emitidas por los sujetos con respecto al tamaño real de los estímulos presentados.

Ahora bien, el interés de los autores parece centrarse en torno a la exactitud de las respuestas, es decir, en si éstas son o no correctas; perdiendo interés los procesos cognitivos realizados por el sujeto para elaborar su estimación. Ello explica que se mantenga constante el ángulo visual en todas las situaciones experimen- 
tales. Pero, además, al no pretender, primordialmente al menos, accionar los mecanismos propios de la constancia perceptiva del tamaño, los resultados obtenidos son más bien estimaciones sobre el tamaño de los objetos que sobre la constancia de los mismos.

La segunda orientación, de tendencia cognitiva y constructivista (Bermejo, I $98 \mathrm{r}$ a; Denis-Prinzhorn, I961; Lambercier, I956, Piaget, 1961; Kubzansky y otros, I 971 , etc.), se ocupa principalmente de los procesos cognitivos responsables de las conductas de constancia y, en esta óptica, la ocurrencia de que las respuestas sean correctas o exactas puede resultar trivial, si no proceden directamente de la activación del proceso que se desea estudiar. Por ello, presentamos en el trabajo anteriormente mencionado un paradigma alternativo al modelo boringiano, que, a nuestro juicio, resulta más apropiado para activar y, en consecuencia, para estudiar los procesos perceptivos peculiares de la constancia visual del tamaño. Con este objetivo de captar el funcionamiento propio de la constancia, nuestro paradigma hace variar sistemáticamente el ángulo visual o tamaño de la imagen retiniana del observador, en conformidad con el concepto comúnmente aceptado por los autores sobre constancia perceptiva del tamaño, descrito ya por Descartes en su «Dioptrique» (1637). De este modo, aunque el ángulo visual formado por los objetos-estímulos varíe en función de la distancia existente entre el ojo del observador y estos mismos objetos, no obstante, el tamaño o dimensiones de tales objetos se consideran como prácticamente invariables, dando lugar a la constancia de los mismos (para más detalles, Bermejo, I98 I b).

La tercera orientación está formada por un pequeño grupo de autores (Epstein, 1977; Gregory, 1963, 1966; Gruber, 1954; Leibowitz y otros, 1978) que recogen aspectos distintos pertenecientes a las dos posiciones comentadas anteriormente. Asi, por ejemplo, Gruber (1954) sigue, a nuestro entender, el paradigma boringiano desde el punto de vista metodológico, como puede constatarse en la con- signa fenomenista propuesta a los observadores; mientras que en el análisis e interpretación de los datos obtenidos toma como referencia el modelo cognitivo. Lo mismo cabe decir con respecto a Gregory (1963), en el sentido de que este autor presenta una definición de la constancia en términos cartesianos, mientras que pretende ilustrarla mediante un ejemplo peculiar de la ley de Emmert.

La adopción de presupuestos epistemológicos diferentes conlleva no sólo a la utilización de paradigmas distintos, como acabamos de ver, sino también en la obtención de datos empíricos diversos. Desde esta perspectiva, no es extraño que algunos autores encuentren empíricamente que el niño pequeño adquiere la constancia visual del tamaño hacia los seis meses (Cruikshank, I 94I; Day y McKenzie, 1981; McKenzie, 1976, McKenzie, Tootell y Day, 1980); mientras que otros sostienen una mayor precocidad, llegando incluso a considerarla innata (Bower, 1965, 1978, 1979; Traverthen y otros, 1975). Y lo mismo acontece con respecto a la evolución con la edad de este feriómeno perceptivo, de modo que la mayor parte de autores propugnan esta evolución (Bermejo, 1981, a; Beyrl, 1926; Denis-Prinzhorn, 1961; Kubzansky y otros, 1971; Lambercier, 1946; Leibowitz y otros, 1967 ; Piaget, 1961 ; Smith y Smith, I 966; Wohlwill, I960, I970); mientras que otros niegan su existencia (Akishige, 1935; Makino, 1965; Rapoport, I969; Teghtsoonian y Beckwith, 1976; Tronik y Hershenson, 1979).

\section{La permanencia del objeto}

Un segundo invariante cognitivo que aparece poco después, según la mayoría de los especialistas, en el desarrollo del niño pequeño se refiere a la noción de permanencia del objeto, sea éste físico o social (la persona humana). Sin entrar aquí en los detalles de una posible distinción en la adquisición de ambos objetos, queremos señalar que ya Piaget en 1937 , relata que Lucienne, su hija menor, llegaba a descubrir a su padre a los ocho 


\section{Estudios}

meses, cuando se escondía detrás de una manta; mientras que a esta misma edad se mostraba incapaz de encontrar un objeto cualquiera ubicado tras una pantalla. Esta disparidad cronológica ha sido confirmada posteriomente desde el punto de vista empírico por varios autores (Bell, 1970; Chazan, I981; Gouin-Decarie y Ricard, 1983; Jackson, Campos y Fisher, 1978; Lamb, I973); no obstante, parece probable que esta diferencia temporal no sea suficiente apoyo para hablar de desfases horizontales, sino que más bien constituirían simples «asincronismos intranocionales", como los denominan Pinard y Laurendau ( 197 I).

Ciñéndonos principalmente al estudio de la permanencia del objeto físico, la abundante bibliografía en torno a este tema no ha sido suficiente razón para alcanzar el consenso entre los autores (Bower, 1974, 1979; Bower y col., 1971, 1972; Butterworth, I98 I; Butterworth y Jarret, 1982; Chromiak y Weisberg, I981; Cornell, 1978; Freeman y otros, 1980; Gratch, I 977; Gratch y otros, I 974; Harris, 1975; Lingle y Lingle, I98 I, etc.). $\mathrm{Y}$ una de las razones fundamentales de este desacuerdo reside, a nuestro juicio, aparte posibles y frecuentes discrepancias metodológicas, en la adopción de niveles distintos de análisis del procesamiento de información; de modo que la noción de permanencia del objeto recubriría procesos cognitivos distintos, en conformidad con la orientación seguida por los distintos autores. En esta perspectiva, resulta obvio que la mera discriminación del objeto supone por parte del niño el poner en juego procesos relativamente simples, como parece acontecer tanto en la discriminación visual (Bower, 1978, 1979; Carpenter, 1974; Fagan, 1970; Fantz, 1961; Fantz y otros, I975; Ruff, I982; etc.), que llegaría a alcanzar un desarrollo notable hacia los cinco meses de la vida del niño (Olson, 1981); como en la discriminación auditiva (Kearsley, 1973; Morse y Cowan, 1982; Wertheimer, 196r; Wishart y otros, 1978), gustativa (Johnson y Salisbury, 1975; Lipsitt y col., 1974, 1976), táctil (Clarkson y Berg, 1977; Pomerleau, Malcuit y Clifton, 1975) y olfativa (Engen,
Lipsitt y Peck, 1974; Rovee, 1972), que presentan un desarrollo sorprendentemente precoz. Así, por ejemplo, MacFarlane (1975, 1977) encuentra con respecto a la discriminación olfativa, que el niño de seis días distingue el olor de la leche materna del olor lácteo de otras mujeres.

Igualmente, la identificación constituye un paso más en la formación de la noción de permanencia del objeto. El niño llega a comprender que a pesar de las diferentes transformaciones espaciotemporales experimentadas por un objeto, éste conserva su propia identidad. No es momento para analizar los distintos procesos implicados en la identificación, desde el punto de vista evolutivo, por lo que remitimos al lector interesado a los notables trabajos de Vurpillot (ver, por ejemplo, 1976). Baste señalar para los objetivos de este estudio que se trata de un proceso más complejo que el supuesto por la mera discriminación, implicando un nivel de procesamiento más elaborado y profundo (Bower, 1978, 1979; Butterworth, I 975, 198I; Butterworth y otros, 1981; Harris, 1982; Vurpillot, 1976). En este sentido precisa Butterworth (1981, pág. 162) de modo conciso pero claro: «infants at stage IV perceive invariance but do not conceive of itm.

La permanencia del objeto, tal como la concibe Piaget (1937), requiere aún la presencia de procesos cognitivos más complejos. Partiendo del presupuesto epistemológico de que en un principio el niño vive sumido en un profundo adualismo con su entorno y de la existencia de una estrecha interrelación a lo largo de desarrollo de las diferentes nociones sensorimotoras (objeto, espacio, causalidad, etcétera), la concepción pagetiana sobre la permanencia del objeto supone necesariamente la localización del mencionado objeto (que a su vez implica, al menos, la construcción sincrónica de las nociones de espacio, tiempo y causalidad) y la búsqueda activa del mismo por parte del niño. Ahora bien, esta conducta de búsqueda sólo puede aparecer lógicamente cuando se haya constituido un dualismo claro, mediante la construcción tanto del yo personal, distino e independiente (aun- 
que relacionado) de su entorno, como del espacio vital en el que discurre habitualmente la actividad del niño.

Esta concepción "fuerte» de la noción de objeto conlleva a situar su aparición en el desarrollo más bien tardíamente, alrededor de los ocho meses, momento en que se inicia el cuarto estadio sensorimotor (Piaget, 1937). Sin embargo, no pocos autores han criticado la posición piagetiana, abogando por una mayor precocidad en la aparición de esta noción (Bower, 1974, 1979; Bower y otros, 1971; Freeman y otros, I980; Gratch, I975; Harris, I 982 ; Moore, I 975 ; Moore y otros, 1978). Los trabajos de Bower y colaboradores principalmente se han presentado como alternativa a la ya prístina posición piagetiana. No es tarea de este trabajo emprender un análisis detallado de las diferencias y semejanzas posibles entre ambas opciones. El lector castellano tiene a su alcance la obra de Bower Development in infancy (1974), traducida con el título El desarrollo del niño pequeño (1979), que recoge los principales experimentos realizados por este prestigioso grupo de investigadores de la Universidad de Edimburgo. Señalemos solamente que los hábiles experimentos elaborados por el equipo de Bower se sitúan, a nuestro juicio, en el ámbito de la percepción, de modo que este mismo autor intitula el capítulo dedicado al estudio del objeto en el libro mencionado anteriormente «la percepción del objeto». En cambio, en la óptica piagetiana se trata de la noción de objeto, que razonablemente supone procesos cognitivos más complejos. Por ello, no es extraño que surjan discrepancias temporales, cuando existen de hecho diferencias de naturaleza. Así se confirma, por otra parte, en las palabras de Butterworth (1981, pág. 196): «it has been argued here that perception of object permanence may be possible before the object concept is acquired».

\section{LA NOCION DE CONSERVACION}

El apartado anterior pretende primordialmente servir de contexto teórico-evo- lutivo para facilitar el estudio y comprensión de la noción de conservación. Como apuntábamos en la Introducción, las conservaciones constituyen uno de los temas que más páginas han acaparado en la literatura psicológica y, sin embargo, sigue siendo aún un área controvertida. Nuestra modesta contribución en las líneas que siguen pretende fundamentalmente dilucidar presupuestos paradigmáticos y epistemológicos y fijar posiciones conceptuales. Esta tarea resultará probablemente menos ardua y laboriosa a la luz del análisis realizado en el estudio de los anteriores invariantes cognitivos.

\section{Precocidad e identidad}

Uno de los puntos de desacuerdo más notorio, tomado el término en su sentido etimológico de visibilidad manifiesta, se refiere al momento de adquisición de dicha noción en el desarrollo infantil. $Y$ si bien este problema no presenta per se mayor interés en la investigación evolutiva, tal como apuntamos en otro estudio dedicado expresamente al análisis de la función de la edad en Psicología evolutiva (Bermejo, 1982 b); puede, no obstante, ocultar la presencia de profundas discrepancias que afecten a la naturaleza misma de la noción examinada.

Todos o la mayor parte de los autores que abogan por la precocidad en la adquisición de la conservación, fundamentan esta noción sobre el principio de identidad (Acredolo, 1981, 1982; Anderson y Cuneo, I978; Brainerd, 1977; Bruner, 1966; Bryant, 1974; Conchillo, 1982 ; Gelman, 1972; Hamel, I971; Pennington y otros, I 980; Shultz y otros, I 979; Siegler, 1981). Así, por ejemplo, Acredolo (1982) transcribe este principio mediante la siguiente "regla de indentidad»: "In the absence of addition or subtraction quantity (amount) is maintained» (pág. 1), y supone que la adquisición de la noción de conservación depende de la comprensión por parte del niño de la regla menciona$\mathrm{da}$, apareciendo ambas sincrónicamente en el desarrollo infantil. En cambio, según este mismo autor, los conceptos de 
compensación y de inversión (las dos formas que puede adoptar la reversibilidad según la escuela de Ginebra) serian comprendidos por el niño en una etapa posterior, caracterizada por la presencia del agrupamiento operatorio y la resistencia a la extinción de la noción de conservación (págs. 22-23).

En consecuencia, Acredolo, al igual que lo hiciera Bruner en 1966, sostiene que el principio de identidad, tal como ha sido formulado anteriormente, constituye la condición necesaria y suficiente para obtener la noción de conservación; de modo que la reversibilidad surgiría como una consecuencia de este principio, y no como causa del mismo (pág. 26).

\section{La conservación operatoria}

la posición piagetiana se muestra una vez más exigente en la conceptualización de las nociones de conservación (Piaget $\mathrm{e}$ Inhelder, I 94 I; Piaget y Szeminska, I 94 I). La identidad perceptiva, recogida en la regla propuesta por Acredolo, resulta claramente insuficiente para fundamentar la noción estudiada; de modo que será necesario la superación del dato perceptivo mediante su operacionalidad, para llegar a adquirir la conservación. Así, por ejemplo, en las tareas de conservación de líquidos, la compensación resulta de la composición operatoria de las variaciones perceptivas referentes a las dimensiones ancho $x$ alto, de manera que el niño puede anular mentalmente las modificaciones percibidas para concluir la necesidad de la invarianza del sistema. En otras palabras, la clave de la noción de conservación piagetiana radica, a nuestro juicio, en la comprensión por parte del niño de la constancia del sistema, a pesar de las múltiples modificaciones, variaciones $u$ operaciones que puedan realizarse con los elementos o partes constitutivas de dicho sistema, siempre que no lleguen a afectar a la totalidad del mismo. De aquí que el paradigma «mágico» de Gelman (1972, 1978), consistente en retirar o añadir un elemento al conjunto cuando el sujeto está distraído, resulte cuestionable debido a la transformación del sistema mismo que se produce, entre otras razones (Silverman y Briga, 1981). Ahora bien, así como la identificación o reconocimiento del objeto resultan insuficientes, según la escuela de Ginebra, para constituir la noción de permanencia del objeto, y el paradigma boringiano es igualmente inapropiado para estudiar las constancias perceptivas, como vimos en el apartado anterior; la constatación perceptiva o intuitiva de la invarianza del sistema se muestra también insuficiente para que el niño adquiera la conservación operatoria. Y la razón fundamental, o al menos una de las razones más importantes, de esta posición «fuerte» de Piaget en la formación de los invariantes cognitivos a lo largo del desarrollo, así como su concepción general del desarrollo infantil mismo, hay que buscarla menos en la Psicología que en las posiciones epistemológicas adoptadas por este autor. A veces se han pasado por lupa los más mínimos detalles de las situaciones empíricas piagetianas (consignas, materiales, procedimientos, etc), discutiendo animosamente distintos componentes de las nociones de conservación (reversibilidad, identidad, equivalencia, necesidad lógica, etc.), sin percatarse, o al menos tener en cuenta, que tanto las características de las situaciones empíricas como los distintos conceptos incidentes en la conceptualización de las conservaciones adquieren su significado peculiar dentro de un marco epistemológico bien determinado.

En consecuencia, desde la óptica evolutiva de esta teoría, el dato perceptivointuitivo, la constatación empírica y la respuesta correcta pueden ser triviales per se, ya que, en el caso de esta última, el objetivo directo de la investigación no se centra en torno a la obtención de respuestas correctas para, por ejemplo, enfatizar la precocidad infantil, sino que más bien persigue el análisis de la contrucción de procesos cognitivos responsables de la noción estudiada. Igualmente, el papel de la constatación empírica en los procesos evolutivos está claramente supeditado a la actividad constructiva del niño. En otras palabras, lo dicho se clarifica a la 
luz de la opción constructivista adoptada por Piaget y que compendiamos en el siguiente principio: «el conocimiento no se copia, sino que se construye».

Pkaget sigue, en parte, tanto la línea marcada por el constructivismo de Berkeley (1709), como la distinción propuesta por Kant entre preceptos y conceptos, y recogida en nuestros dias por Gibson en su teoría perceptiva (Gibson, 1966, 1979). No obstante, si para Kant las categorías básicas de espacio, objeto, tiempo y causalidad son formas a priori, Piaget se distancia de esta posición considerando que tales categorías proceden de la experiencia del sujeto con su entorno, poniendo de relieve el carácter claramente constructivista de su teoría. En esta óptica, los invariantes cognitivos no son categorías kantianas a priori, ni tampoco copias impuestas por el medio ambiente. Se trata, más bien, de construcciones realizadas por el niño en relación con su propio entorno. Por ello, resulta lógico que la concepción piagetiana sobre el tema se muestre más exigente y requiera procesos cognitivos más complejos que la noción basada exclusivamente en el principio de identidad presentado por los autores an teriormente mencionados. Precisamente, el problema fundamental de la conservación uest celui du conflit entre l'expérience immédiate ou les données de la perception, d'une part, et les opérations rationnelles, d'autre part" (Piaget e Inhelder, 1941, pág. I 5); de modo que la noción de conservación, según estos autores, es por naturaleza operatoria, o dicho de otro modo, requiere la presencia de procesos cognitivos que aseguren la invarianza del sistema, a pesar de las posibles transformaciones que puedan acaecer en su interior, como cambios de forma, división en partes, etc. Sintetizando, la conservación es operatoria o no se trata de una auténtica conservación.

Ahora bien, este tipo de conservación postula necesariamente la presencia de operaciones cognitivas que, anulando mentalmente la transformación producida o componiendo adecuadamente determinadas informaciones, conduzca lógicamene a la conclusión de la invarianza.
Requiere, pues, no sólo la presencia de una (o varias) transformación, sino también que el niño llegue a comprender dicha transformación y su respuesta proceda directamente de esta comprensión. Por ello, si el paradigma metodológico empleado no asegura necesariamente la concurrencia de estas tres condiciones, puede ponerse en duda su eficacia para determinar empíricamente la presencia o ausencia de la conservación operatoria en el desarrollo del niño.

\section{Identidad versus conservaciones operatorias}

En sendos trabajos (Bovet, Parrat-Dayan y Deshusses-Addor, 1981 ; Parrat-Dayan y Bovet, 1982), Bovet y colaboradores se plantean el problema de la precocidad de la conservación, analizando detalladamente algunos de los trabajos defensores de la misma. Después de insistir en que, para Piaget, "le problème de la conservation consiste à reconstruire la transformation de manière à pouvoir l'annuler opératoirement» (pág. 290), muestran que el diseño empírico utilizado por Light, Buckingham y Robin (1979) no permite la obtención de respuestas operatorias. Al contrario, las situaciones experimentales presentadas por estos autores parecen buscar primordialmente la producción de «respuestas correctas» por parte de los niños, sin prestar atención, o apenas, a la transformación propiamente dicha.

Lo mismo cabe decir, con ciertas matizaciones, sobre las respuestas empíricas obtenidas en el interesante trabajo de Conchillo (1982), que sobresale por su gran sentido crítico y no menos profundidad a lo largo de sus páginas. La autora pretende principalmente mostrar la eficacia de los métodos no verbales con respecto a la estrategia clásica verbal, abogando también por la precocidad de las nociones de conservación. En ambos casos los resultados empíricos confirman sus objetivos, ya que las pruebas no verbales presentan una proporción de $33 / 85$, mientras que en las verbales la 
proporción desciende considerablemente $(2 / 8 \mathrm{~s})$. Además, el hecho de que 33 niños de edad media 5,2 años (oscilando las edades entre 4,2 y 5,8 ) sobre 85 de la muestra emitan respuestas correctas, permite concluir, con toda lógica, que la noción estudiada parece ser más precoz de lo supuesto por la escuela de Ginebra.

Sin embargo, con respecto al tema de la precocidad quisiéramos hacer dos puntualizaciones (de algún modo sugerencias), que afectan sólo en parte al trabajo comentado. En primer lugar, la variable edad tiene un valor muy relativo como referente o indicador del momento de aparición de cambios conductuales a lo largo del desarrollo (Bermejo, 1982 b), de modo que algunos autores aconsejan más bien utilizar otras variables para organizar $\mathrm{u}$ ordenar los cambios evolutivos, como, por jemplo, los estadios, épocas, el tiempo psicológico, etc. (Thomae, I979; Zazzo, 1967). De hecho, los primeros precursores históricos de la metodología longitudinal (Hollingworth, 1927; Stern, 1910) no emplean la edad cronológica como criterio primario para definir este tipo de estrategia. Y en segundo lugar, sugerimos que podría ser interesante, desde el punto de vista evolutivo, conocer en concreto las edades de los sujetos que respondieron correctamente (esto parece que no se indica en el trabajo), ya que la muestra oscila entre 4,2 y 5,8 años, y esta amplitud de un año y medio puede ser significativa en este momento preciso del desarrollo. No lo sería quizá si las edades estuvieran comprendidas entre 7,6 y 9,0 , ó entre I 5,0 y I6,6, y sobre todo para sujetos adultos de 39,0 y 36,6. En cambio, la respuesta sería con toda seguridad positiva, si se tratara de sujetos de 0,3 a I,9 años, por ejemplo, dependiendo siempre de la dimensión psicológica estudiada. Pero, además, los psicólogos evolutivos conocemos aún poco sobre las peculiaridades propias del desarrollo infantil entre los tres o cuatro años hasta los seis; de modo que algunos autores han llegado incluso a hablar de "anarquía» en el desarrollo de ciertos aspectos cognitivos durante este lapso de tiempo (ver Bideau, 1979), como, por otra parte, parecen apuntar parcialmente nuestros resultados sobre la evolución de las conductas de clasificación, que publicaremos próximamente (Bermejo y col., próxima aparición).

Ahora bien, en cuanto a las diferencias encontradas entre las pruebas no verbales y las verbales nos parecen totalmente justificadas, ya que, a nuestro entender, estas pruebas podrian medir procesos cognitivos diferentes, en el sentido de que las segundas serían apropiadas, según aparecen descritas, para «medir» la conservación operatoria, mientras que las primeras podrían no serlo. En otras palabras, teoría y método suelen ir estrechamente relacionados en todos los ámbitos científicos $y$, en consecuencia, no es extraño que en este caso los dos tipos de pruebas empleadas supongan conceptualizaciones diferentes de las nociones de conservación, como, por otra parte, parece suponer Conchillo (1982). Así, pues, en el ámbito de las pruebas clásicas compartimos la hipótesis de la autora de que en muchos casos la no conservación es «más aparente que real y no refleja sino un modo inapropiado de expresarse» (1982, pág. I 81 ) el niño. La categorización de las respuestas infantiles en el método clínico constituye una tarea compleja, cuyo éxito depende en gran medida de la pericia del investigador. De aquí que Piaget (1926) insista sobre la importancia de la formación de los futuros investigadores, así como sobre las muchas dificultades concretas que presenta el uso de este método. Aún recordamos - sea dicho entre paréntesis - la reiteración del profesorado de Psicología de la Universidad de Ginebra sobre la necesidad de una prolongada práctica para llegar a utilizar adecuadamente y adquirir su máxima eficacia con el método clínico-crítico. Con este fin, el plan de estudios de Psicología en Ginebra prevé la participación anual de todos los estudiantes en investigaciones dirigidas por profesores en colegios y guarderías, a partir del segundo curso de carrera.

Si las pruebas clásicas mencionadas pretenden primordialmente determinar la presencia o ausencia de las conservacio- 
nes operatorias, las pruebas no verbales descritas persiguen más bien la producción de respuestas referidas al principio de identidad. Ello explica, por una parte, el poco interés prestado a la comprensión infantil de la transformación propiamente dicha $y$, por otra, el papel preponderante concedido a la memoria a corto plazo, ya que el criterio último para aceptar la validez de una respuesta es de tipo mnemónico. Es más, resulta indispensable que el sujeto recuerde la procedencia del líquido transformado para poder responder correctamente; de lo contrario, el niño, ni probablemente el adulto, tenga suficiente información para emitir la respuesta adecuada. En este sentido, las pruebas no verbales utilizadas resultan más difíciles que las clásicas al menos en dos puntos: primero, porque el procedimiento empírico se inicia partiendo de una desigualdad entre los líquidos en el caso de las pruebas no verbales; mientras que en las verbales se parte de una equivalencia, que facilita, al menos ligeramente, la tarea de los niños en la continuación de la prueba. Pero, sobre todo, en las pruebas no verbales se producen dos transformaciones, mientras que en las clásicas sólo se realiza una de ellas.

No obstante, los resultados obtenidos con las pruebas no verbales son notoriamente superiores, debido fundamentalmente, a nuestro entender, a que la producción de la respuesta correcta no requiere necesariamente la comprensión infantil de la transformación del líquido, sino sólo el recuerdo de la procedencia del líquido trasvasado. En otras palabras, mientras que las pruebas operatorias se centran en torno a la transformación misma, la prueba no verbal utilizada presta poco interés a las transformaciones, ocupándose principalmente de la referencia de niveles y de la asociación de dos vasos determinados. En consecuencia, las discrepancias entre los resultados obtenidos con los dos tipos de pruebas están sobradamente justificados, aunque no puede negarse la posible presencia de otras variables (lingüísticas, sociales, etc.), que requieren un minucioso control empírico.

\section{CONCLUSIONES}

A lo largo de este trabajo hemos pretendido desvelar y poner de relieve la importancia de los presupuestos epistemológicos y paradigmáticos en la conceptualización de las conservaciones, tanto a nivel de planteamiento de los problemas como referente a la estrategia metodológica utilizada y la interpretación de los datos empíricos obtenidos. Mediante el estudio de algunos trabajos, que nos han servido de referencia, hemos defendido la tesis de que la mayor parte de las discrepancias existentes entre los autores son, en principio, de tipo semántico, en el sentido de que el mismo término o expresión recubre a veces procesos cognitivos diferentes $y$, en consecuencia, implican niveles distintos de procesamiento de la información.

Desde esta perspectiva, el análisis de los estudios más significativos en torno a las nociones de conservación, así como sobre otros invariantes cognitivos más precoces evolutivamente, nos permite pergeñar las conclusiones siguientes: I) Los estudios sobre la constancia visual del tamaño siguen dos tendencias principales: por una parte, se sitúan los trabajos que adoptan como modelo el paradigma boringiano (Boring, 1940; Gilinski, I95I; Halways y Boring, I94I; Kilpatrick e Ittelson, I953; Kuroda, 1963, etc.), mientras que otros autores toman, más bien, una posición cognitiva y constructivista (Bermejo, 1981 a; Denis-Prinzhorn, 1961; Kubzansky y otros, I971; Lambercier, 1956; Piaget, I961, etc.).

2) En cuanto a la permanencia del objeto, también aqui se refleja la misma dicotomía general entre los autores. Por una parte, aparece la concepción "débil» de la noción de permanencia del objeto, representada principalmente por el grupo de investigadores reunidos en torno a Bower, que centran sus trabajos, más bien, sobre la percepción del objeto (Bower, I 974, 1978, 1979; Bower y otros, 1971; Harris, 1982; Moore, I975; Moore y otros, 1978). Y, por otra, la concepción "fuerte" propuesta por Piaget en 1937, y seguida después pot otros autores, a ve- 
ces con matices (Butterworth, $198 \mathrm{I}$; Butterworth, Jarret y Hicks, I982; GouinDecarie y Ricard, 1983; Laurendau y Pinard, 1968), que abogan por criterios más exigentes para determinar la presencia o ausencia de la noción de objeto, como la localización espacial y la búsque. da activa del mismo por parte del niño.

3) Los trabajos sobre las conservaciones muestran igualmente esta duplicidad de tendencias: unos fundamenan esta noción sobre el principio de identidad, defendiendo su aparición precoz en el desarrollo infantil (Acredolo, 1981, 1982; Anderson y Cuneo, 1978; Bruner, 1966; Bryant, 1974; Conchillo, 1982 , 1983 ; Pennington y otros, 1980; Shultz y otros, 1979; Siegler, 1981), mientras que otros suponen la presencia de procesos cognitivos más complejos, como la escuela de Ginebra que propone la conservación operatoria (Piaget e Inhelder, 1941, 1963;
Piaget y Szeminska, I 94 I; Bovet, ParratDayan y Deshusses-Addor, 1981; ParratDayan y Bovet, I982, etc.).

4) Finalmente, esta disparidad de líneas seguidas por los trabajos publicados sobre los distintos invariantes cognitivos jalonados a lo largo del desarrollo resulta sumamente instructiva, no sólo porque facilita la comprensión del debatido tema de las conservaciones, al situar éstas en su contexto evolutivo peculiar, sino porque, además, manifiesta la adopción de tendencias epistemológicas diferentes, determinantes, al menos en parte, de las posiciones concretas defendidas por los autores. La dificultad (sumamente atractiva) aparece cuando se pretende conjugar o integrar ambas orientaciones en un mismo sistema teórico, mientras que surge el error cuando se inenta refutar o defender una orientación con datos empíricos obtenidos a partir del otro paradigma.

\section{Referencias}

ACREDOLO, C.: "The acquisition of conservation: A clarification of Piagetian terminology, some recent findings, and an alternative formulation". Human Devel, 1981, 24, $120-137$

ACREDOLO, C.: «Conservation-Nonconservation: Alternative explanations». En Brainerd, Ch. J. (Ed.): Children's logical and matbematical cognition, N. York: Springer-Verlag, 1982, 1-31.

Akishige, Y: «Experimental studies of the structure of perceptual space (IV): Developmental problems of perceptual constancies». Test-sugaku.Sbigaku-Bungaku Rombunsbu, Tokyo: Iwanani, 1937, 467-570.

ANDERSON, N. H. y CUNEO, D. O.: "The height + width rule in children's judgement of quantity". J. of Exp. Psychol., 1978, 107, 335-378.

BEI.I, S. M.: "The development of the concept of object as related to infant-mother attachement». Child Dev., 1970, 4I, 291-3II.

BERMEjO, V.: Fenómenos perceptivos normales y deformantes. La constancia y las ilusiones. Barcelona: Ed. Univ. Autónoma de Barcelona, i981a.

BERMEJO, V.: «Investigaciones sobre la constancia visual del tamaño. Tendencias y estrategias experimentales». Anuario de Psicologia, $198 \mathrm{Ib}, 24,58-83$.

Bermejo, V.: "Desarrollo cognitivo". Psiquis, 1982a, 3, 12-39.

BERMEjO, V.: «El concepto de cambio y la función de la edad en Psicología evolutiva». Informes de Psicol., $1982 \mathrm{~b}, 3,15-34$.

BEYRL, F.: "Über die Graossenauffassung bei Kindern». Z. $f$. Psychol., 1926, 100, 344-371.

Bideau, J.: «Etude génétique des conduites verbales et pratiques de classification». Bul. de Psychol, 1979, $32,565-576$.

Boring, E.: «Size constancy and Emmert's law». Amer. J. Psychol., i 940, 53, 293-295.

Bovet, M.; Parrat-Dayan, S., y Deshusses-Addor, D.: «Peut-on parler de précocité et de regression dans la conservation? I. Précocité». Arch. de Psychol., 1981, 49, 289-303.

BOWER, T. G. R.: «Stimulus variables determining space perception in infants». Science, 1965, 149, 88-89.

Bower, T. G. R.: Development in infancy, San Francisco: Freeman and Company, 1974.

BOWER, T. G. R.: Human development, San Francisco: Freeman and Company, 1979.

Bower, T. G. R.; Broughton, J. M., y Moore, M. K.: "The development of the object concept as manifested by changes in the trancking behavior of infants between 7 and 20 weeks of agem. $J$. Exp. Cbild Psychol., $1971,11,2$.

Bower, T. G. R., y PAterson, J. G.: «Stages in the development of the object concept». Cognition, 1972, $I, 47^{-55}$. 
BrainerD, C. J.: «Feedback, rule, knowledge and conservation learning». Child Dev., 1977, 48, 404-41 I.

BRAINERD, C. J.: «Markovian interpretations of conservation learning". Psychol. Review, 1979, $83,181-213$.

BRAINERD, C. J.: «Investigaciones sobre aprendizaje y la teoria de Piaget». En Siegel y Brainerd (Eds.): Alternativas a Piaget, Madrid: Pirámide, 1982, 83-1 20.

BRUNER, J. S.: «On the conservation of liquids». En Bruner, Olver y Greenfield (Eds.): Studies in cognitive growth, N. York: Wiley, 1966.

BRYANT, P. E.: Perception and understanding in young children: An experimental approach, N. York: Basic Books, 1974 .

BUTTERWORTH, G. E.: "Object identity in infancy: The interaction of spatial location codes in determining search errorm. Child Dev., 1975, 46, 866-870.

BUTTERWORTH, G. E.: "Object permanence and identity in Piaget's theory of infant cognition". En Butterworth, G. E. (Fd.): Infancy and epistemology, Sussex: The Harvester Press, i981, $175-185$.

Butterworth, G. E., y JARRET, N.: «Piaget's stage IV error: background to the problem». Brit. J. of Psycbol., 1982,73, 175-185.

CARPENTER, G.: «Mother's face and the newborn». New Scientist, 1974, mars, 742-744.

Conchillo, A.: Medida de la noción de conservación de la cantidad, Madrid: Ed. Univ. Complutense, i982.

Conchillo, A.: "La reversibilidad como clave de la conservación: ¿realidad o necesidad? Informes de Psicologia, 1983,2, 221-231.

CORNELI., E. H.: "Aprendiendo a encontrar cosas: una reinterpretación de los estudios sobre la permanencia del objeto». En Siegel y Brainerd (Eds.), i 982.

COWAN, R.: "Performance in number conservation tasks as a function of the number of items». Brit. J. of Psycbol., I 979, 70, 77-8 I.

Cruikshank, R. M.: "The development of visual size constancy in early infancy». J. Genet. Psychol., I941, $58,327-351$.

ChazAN, S. E.: «Development of object permanence as a correlate of dimensions of maternal care». Dev. Psychol., 1981, 17, 79-81.

Chromiak, W. y WeIsberG, R. W.: "The role of the object concept in visual tracking: child-like errors in adults". J. of Exp. Child Psychol., $1981,32,531-543$.

DAY, R. H., y MCKENZIE, B. E.: "Infant perception of the invariant size of approaching and receding objects». Dev. Psychol., 1981, 17, 670-677.

Denis-Prinzhorn, M.: Perception de distances et constances de grandeurs, Genève: These de doctorat, 1961 .

Descartes, R.: Dioptrique, París: Gallimard, 1953.

Donaldson, M.: «Conservation: What is the question? Brit. J. of Psychol., 1982, 73, 199-207.

EMmert, E.: «Grössenverhältnisse der Nachbilder». Klin. monatb. Augenbe, i 88 1, 443-450.

ENGEN, T.: LIPSITT, L. P., y PECK, M. B.: «Ability of newborn infants to discriminate sapid substances». Dev. Psychol., 1974, 10, 741-744.

EPSTEIN, W.: «Historical introduction to the constancies». En Epstein, W. (Ed.). Stability and constancy in visual perception: mechanisms and processes, N. York: Wiley, 1977, 1-22.

FAGAN, J. F.: «Memory in the infant». J. of Exp. Child Psychol., 1970, 9, $217^{-226 .}$

FANTZ, R. L.: "The origin of form perception». Scient. Amer., 1961, 204, 66-72.

FANTZ, R. L.; Fagan, J. F., y MiRanda, S. B.: "Early visual selectivity". En Cohen, L. B. y Salaparek (Eds.): Infant perception: From sensation to cognition, N. York: Academic, 1979.

Freeman, N. H.; LloYd, S., y SiNHA, G. G.: "Infant search tasks reveal early concepts of containment and canonical usage of objects". Cognition, 1980, 8, 243-262.

GELMAN, R.: «Logical capacity of very young children: Number variance rules». Cbild Dev., 1972, 43, 75-90.

Gelman, R.: "Cognitive development». Ann. Rev. Psychol., 1978, 29, 297-332.

Gelman, R.: «What young children know about numbers». Edwc. Psycbologist, 1980, 15, 54-68.

GiBson, J. J.: The ecological approach to visual perception. Boston, Massachusetts: Houghton Mifflin, 1979.

GilinSKI, A.: «Perceived size and distance in visual space». Psychol. Rev., I95 I, 58, 460-482.

Gouin Decarie, T., y Richard, M.: “La socialización del lactante». Mundo cientifico, 1983, 22, febrero, I $20-129$.

Gratch, G.: Recent studies based on Piaget's view of object concept development. En Cohen y Salapatek (Eds.), 1975.

GRATCH, G.: "Review of piagetian infancy research. Object concept development". En Overton y Gallagher (Eds.). Knowledge and development, Vol. I, N. York: Plenum Press, 1977, 59-91.

Gratch, G.; ApPel, K. J.; Evans, W. F.; LeCompte, G. K., y Wright, N. A.: «Piaget's stage IV object concept error: Evidence of forgetting or object conception». Child Dev., 1974, 45, 71-77.

Gregory, R.: «Distortion of visual space as inappropiate constancy scale». Nature, 1963, 199, 678-680.

GREGORY, R.: Eye and brain. Londres: Weidenfeld \& Nicolson, 1966.

Gruber, H.: "The relation of perceived size to perceived distance». Amer. J. Psychol., 1954, 67, 411-426.

HAlways, A., y Boring, E.: "Determinants of apparent visual size with distance variant". Amer. $J$. Psychol., 1941, 54, 21-37.

HAMEL, B. R.: "On the conservation of liquids". Human Dev., 1971, 14, 39-46.

Hargreaves, D. J.; Molioy, C. G., y PRATT, A. R.: "Social factors in conservation». Brit. J. of Psychol., $1982,73,231-234$.

Harris, P. L.: «Development of search and object permanence during infancy». Psychol. Bul., 1975, 82, $332-344$.

HuNT, T. D.: "Early number "conservation" and experimenter expectancy". Child Dev., 1975, 46, 984-987. 
INHELDER, B.: «Observations sur le principe de conservation dans la physique de l'enfant». Cabiers de Pédagogie Expérimentale et de Psycbologie de l'Enfant, 1936, 9 .

KEARSLEY, R. B.: "The newborn's response to auditory stimulation: a demostration of orienting and defensive behavior». Child Dev., 1973, 44, 582-590.

KanNo, Y.: «Conservation, transitivity and class inclusion of number». Toboleu Psychologica Folia, 1979, 38 , 8-17.

KilPATRICK, F. P., e Itelson, W. H.: «The size distance invariance hypothesis». Psychol. Rev., 1953, 60, $223-231$.

KubZanSky, Ph.; Rebeisky, F., y DoRman, L.: «A developmental study of size constancy for two versus three-dimensional stimulis. Child Dev., 1971, 42, 633-635.

KuroDA, T.: «Methodological and experimental studies on size constancy». Japanese Psychol. Research, 1963, S, 188-202.

LAMB, M. E. (Ed.): The role of the father in child development. N. York: Wiley, 1976.

LAMBERCIER, M.: "Recherches sur le développement des perceptions. VII. La configuration en profondeur dans la constance des grandeurs». Arch. de Psychol., 1946, 31, 1-204.

Laurendeau, M., y PINARD, A.: Les premières notions spatiales de l'enfant. Neuchàtel: Delachaux et Niestlé, ig68.

Leibowitz, H. W.; POLlard, S. W., y Dickson, D.: "Monocular and binocular size matching as a function of distance at various age levels». Amer. J. Psycbol., 1967, 80, 263-268.

Light, P. H.; Buckingham, N. y Robbins, A. H.: "The conservation task as an interactional setting". Brit. J. of Educ. Psycbol., 1979, 49, 304-310.

Lingi.E, K. M., y LiNGLE, J. H.: "Effects of selected object characteristics on object-permanece test performances. Cbild Dev., 1981, 52, 367-369.

LIPSiTT, L. P., y KAYE, H.: «Conditioned sucking in the human newborn». Psycbonomic Science, I974, 1, 29-30.

LIPSITT, L. P.; KAYE, H., y BOSACK, T. N.: «Enhancement of neonatal sucking through reinforcement". J. Exp. Psychol., 1976, 4, 153-168.

MacFarlane, J. A.: Psicología del nacimiento. Madrid: Morata, 1978.

MAKINO, T.: «Developmental problems of size constancy». J. Child Dev., 1965, 1, $31-37$.

MCGarRigi.e, J., y DONAIDSON, M.: “Conservation accidents». Cognition, 1976, 3, 341-350.

MCKenziE, B.: «Perceptual constancies in early infancy». Inter. Cogn. of Psychol., XXI, Paris, 1976, july.

MCKenzie, B.; ToOtell., H. E., y DAY, R. H.: «Development of visual size constancy during the last year of human infancy". Dev. Psychol., 1980, 16, 163-174.

Mul.eER, S. A.: "On the generalizability of conservation: A comparison of different kinds of transformations. Brit. J. of Psycbol., 1982, 73, $22 \mathrm{I}-230$.

MOORE, M. K.: «Object permanence and object identity: A stage-developmental model». Soc. Res. Child Dev., Denver, Colorado, 1975 .

MoOre, M. K.; BORTON, R., y DARBY, B. L.: "Visual tracking in young infants: Evidence for object identity or object permanence?» $J$. Exp. Child Psychol., 1978, 25, $183-197$.

Morse, Ph. A., y Cowan, N.: «lnfant auditory and speech perception». En Field; Huston; Quay; Troll y Finley (Eds.): Revien of buman development, N. York: Wiley, 1982, 32-61.

MURRAY, F. B.: "The conservation paradigm: The conservation of conservation research". En Sigel; Brodzinsky y Golinkoff (Eds.): New directions in piagetian theory and practice, Hillsdale, New Jersey: Lawrence Erlbaum As., 1981, 143-175.

Parrat-Dayan, S., y Bovet, M.: «Peut-on parler de précocité et de regression dans la conservation? II." Arch. de Psycbol, 1982, 50, 237-249.

Pennington, B. F.; Wallach, L., y Wallach, M. A.: «Nonconservers' use and understanding of number and arithmetic». Genet. Psycbol. Monog., 1980, 101, 23 1-243.

PlAGET, J.: Le jugement et le raisonnement cheq l'enfant. Neuchâtel, Delachaux \& Niestlé, 1926.

Plaget, J.: La construction du réel chę l'enfant. Neuchâtel: Delachaux \& Niestlé, 1937.

PIAGET, J.: Les mécanismes perceptifs, Paris: P. U. F., 1961 .

Plaget, J. e InHelder, B.: Le développement des quantitiés chez l'enfant. Conservation et atomisme. Neuchâtel: Delachaux \& Niestlé, $194 \mathrm{I}$.

Piaget, J. e Inhelder, B.: "Les opérations intellectuelles et leur développement». En Fraisse y Piaget (Eds.): Traité de Psychologie Expérimentale. VII: L'intelligence, Paris: P. U. F., 1963.

Pinget, J. y Szeminski, A.: La genèse du nombre chę l'enfant. Neuchâtel: Delachaux \& Niestlé, $194 \mathrm{t}$.

POMERI,AU, A., y CLIFTON, R.: "Neonatal heart-rate response to tactile, auditory and vestibular stimulation in different states". Child Dev., 1973, 44, 48,-496.

RAPOPORT, J.: "Size-constancy in children measures by a functional size-discrimination task". J. Child Psychol., $1969,7,366-373$.

RoveE, C. K.: "Olfactory cross-adaptation and facilitation in human neonates». J. of Exp. Child Psychol., $1972,13,368-381$.

RuFF, H. A.: The development of object perception in infancy. En Field; Huston; Quay; Troll y Finley (Eds.), $1982,93-106$.

RUSSEI.I., J.: "Nonverbal and verbal judgements of length invariance by young children». Brit. J. Psycbol., $1979,70,313-317$.

Shultz, T. R.; DOVER, A., y AmSEL, E.: "The logical and empirical bases of conservation judgement». Cognition, 1979, 7, 99-123. 
SIEGLER, R. S.: «Developmental sequences within and between concepts». Monog. of Soc. for Res. Child Dev., I $981,46,189$.

SIVERMAN, I. W., y BRIGA, J.: "By what process do young children solve small number conservation problems». J. of Exp. Child Psychol., 1981, 32, 11 5-126.

Silverman, I. W., y ROSE, A. P.: «Compensation and conservation». Psychol. Bul., 1982.

SmITH, O. W., y SMITH, P. C.: «Developmental studies of spacial judgements by children and adults». Perc. and Mot. Skills, 1966, 22, 3-73.

TEGHTSOONIAN, M., y BECKWITH, J. B.: "Children's size judgements when size and distance vary: Is there developmental trend to overconstancy?" J. of Exp. Child Psycbol., 1976, 22, 23-39.

Thomas, H.: "The concept of development and life-span developmental psychology". En Baltes y Brim (Eds.): Life-span development and bebavior, Vol. 2, N. York: Academic Press, 1979.

Traverthen, C.; Penelope, H., y Sheeran, L.: «Activités innées du nourrison». Recherche, i975, 28.

Tronick, E., y Hershenson, M.: “Size-distance perception in preschool children». J. of Exp. Child Psychol., 1979, 27, 166-184.

VURPILI.OT, E.: «Development of identification of objects». En Hamilston y Vernos (Eds.): The development of cognitive processes, N. York: Academic Press, 1976, 191-236.

WERTHEIMER, M.: «Psycho-motor coordination of auditory-visual space at birth». Science, 1961, 134, $1692-1696$.

Wheidali., K., y PoborCA, B.: «Conservation without conservation? An alternative, non-verbal paradigm for assessing conservation of liquid quantity». Brit. J. of Psycbol., 1980, 7I, 1 1 7-1 34.

WoHlwil.., J. F.: «Development studies of perception». Psychol. Bul., 1960, 57, 249-288.

WoHıwil., J. F.: «Perceptual development». En Reese y Lipsitt (Eds.): Experimental child psychology, N. York: Academic Press, 1970.

ZAzzo, R.: Conduites et conscience, Neuchâtel: Delachaux \& Niestlé, 1967.

\section{Resumen}

Se analizan primeramente algunos invariantes cognitivos precoces en el desarrollo (la constancia visual del tamaño y la permanencia del objeto), para facilitar el estudio de las conservaciones en un marco conceptual y evolutivo apropiado. La dicotomia de tendencias existente entre los autores sobre esta noción de conservación se refleja igualmente en los invariantes cognitivos anteriores, debido principalmente a la presencia de determinados presupuestos epistemológicos. Concluye el trabajo defendiendo la tesis de que las discrepancias son, en principio, de carácter semántico, en el sentido de que los mismos invariantes recubririan, según los autores, procesos cognitivos diferentes, implicando niveles distintos de procesamiento de información.

\section{Summary}

First, some of development's precocious cognitive invariants (visual constancy of size and object permanency) are analysed in order to facilitate the stydy of conservations in an adequate conceptual and evolutive frame. The dicbotomy of tendencies in different authors about this notion of conservation is equally reflected in the former cognitive invariants, due principally to the presence of certain epistemological assumptions. The work ends by defending the thesis that discrepancies are, in principle, of semantic quality, in the sense that the same invariants might cover, according to the autbors, different cognitive processes, thus implying different levels of information process.

Departamento de Psicologia Evolutiva y Diferencial/Facultad de Psicologia/Universidad Complutense/Campus de Somosaguas/Madrid-23.

\section{Résumé}

On analyse d'abord quelques invariants cognitifs précoces dans le développement (la constance de la grandeur et la permanence de l'objet) pour faciliter l'étude des conservations dans un cadre conceptuel et évolutif aproprié. La dychotomie de tendences actuelle entre les auteurs sur cette notion de conservation se reflète de la même fafon dans les invariants cognitifs antérieurs, ceci étant du principalement à la présence de certaines suppositions épistémologiques. Finalement ce travail défend la thèse que les différents sont, en principe, d'ordre sémantique, en ce sens que les mêmes invariants pourraient couvrir, suivant les auteurs, des processus cognitifs différents, implicant donc divers niveaux de traitement de linformation. 